\title{
Pisot Numbers in the Neighborhood of a Limit Point. II
}

\author{
By David W. Boyd*
}

\begin{abstract}
Let $S$ denote the set of real algebraic integers greater than one, all of whose other conjugates lie within the unit circle. In an earlier paper, we introduced the notion of "width" of a limit point $\alpha$ of $S$ and showed that, if the width of $\alpha$ is smaller than $1.28 \ldots$ then there is an algorithm for determining all members of $S$ in a neighborhood of $\alpha$. Recently, we introduced the "derived tree" in order to deal with limit points of greater width. Here, we apply these ideas to the study of the limit point $\alpha_{3}$, the zero of $z^{4}-2 z^{3}+z-1$ outside the unit circle. We determine the smallest neighborhood $\theta_{1}<\alpha_{3}<\theta_{2}$ of $\alpha_{3}$ in which all elements of $S$ other than $\alpha_{3}$ satisfy one of the equations $z^{n}\left(z^{4}-2 z^{3}+z-1\right) \pm A(z)=0$, where $A(z)$ is one of $z^{3}-z^{2}+1, z^{3}-z+1$ or $z^{4}-z^{3}+z-1$. The endpoints $\theta_{1}$ and $\theta_{2}$ are elements of $S$ of degrees 23 and 42, respectively.
\end{abstract}

1. Introduction. As usual, let $S$ denote the set of Pisot (Pisot-Vijayaraghavan) numbers. In an earlier paper [1], we gave an algorithm for determining all elements of $S$ in a given interval of the real line provided there are only a finite number of elements of $S$ in this interval. We also showed how to determine all elements of $S$ in the neighborhood of certain limit points.

We demonstrated the algorithm by finding all points of $S$ in $[1,1.86675]$ and $[1.868,1.932]$, intervals containing, respectively, three and two limit points of $S$. The reason for the gap $(1.86675,1.868)$ is the presence of the limit point $\alpha_{3}=$ 1.8667603992, the zero of $x^{4}-2 x^{3}+x-1$ in $|x|>1$. In the terminology of [1], this limit point has width $1.7548 \ldots$ and hence cannot be dealt with by the methods of [1].

In the first paper of this series [2], we showed how to extend the algorithm of [1] to deal with limit points such as $\alpha_{3}$. The basic new idea is that of the derived tree. Briefly, each Pisot number $\theta$ is associated with a certain set of rational functions $f=A / Q=u_{0}+u_{1} z+\cdots$ with integer coefficients. The set $\mathscr{C}$ consists of all such $f$ as $\theta$ varies over $S$. The sequences of coefficients $\left\{u_{k}\right\}$ are paths to infinity in a tree $\mathscr{T}$ defined by the inequalities of Schur's algorithm. An $N$-neighborhood of $f$ in $\mathscr{C}$ consists of all $g$ in $\mathscr{C}$ whose first $N+1$ coefficients $u_{0}, \ldots, u_{N}$ agree with those of $f$. If $f$ is a limit point of $\mathscr{C}$ in the topology defined by these neighborhoods then, at each level $n$ of the corresponding path in $\mathscr{T}$, a subtree $\mathscr{T}_{n}(f)$ branches off. The derived tree $\mathscr{T}^{\prime}(f)$ describes the asymptotic behavior of $\mathscr{T}_{n}(f)$ as $n \rightarrow \infty$.

Received August 24, 1983.

1980 Mathematics Subject Classification. Primary 12A15, 12-04.

* Supported in part by the Natural Sciences and Engineering Research Council of Canada.

(C)1984 American Mathematical Society $0025-5718 / 84 \$ 1.00+\$ .25$ per page 
If $\mathscr{T}^{\prime}(f)$ is essentially finite (see [2] for this and other undefined terminology), there is an effective constant $N$ such that all $g$ in the $N$-neighborhood of $f$ can be completely determined. Under quite general conditions [2, Theorem 8.5], this $\mathrm{N}$ neighborhood consists of $f(z)=A(z) / Q(z)$ and the functions

$$
\frac{A(z) \pm z^{n+r} Q\left(z^{-1}\right)}{Q(z) \pm z^{n+r} A\left(z^{-1}\right)}
$$

for $n \geqslant N$, where $r \leqslant \max (\operatorname{deg} A, \operatorname{deg} Q)$ is a certain integer depending on $f$.

Our purpose here is to demonstrate the practicality of the method of [2] by filling in the gap $(1.86675,1.868)$ around $\alpha_{3}$. There are three limit points of $\mathscr{C}$ associated with $\alpha_{3}$, namely

$$
\begin{aligned}
& g_{1,3}=\left(1-z+z^{3}\right) /\left(1-2 z+z^{3}-z^{4}\right), \\
& g_{2,3}=\left(1-z^{2}+z^{3}\right) /\left(1-2 z+z^{3}-z^{4}\right), \\
& g_{3,3}=\left(1-z+z^{3}-z^{4}\right) /\left(1-2 z+z^{3}-z^{4}\right) .
\end{aligned}
$$

The first two of these have width 1 and were already treated in [1]. Thus we can confine ourselves to $g_{3,3}$ which we discussed briefly in [2].

We will show that the only $f$ in the 22-neighborhood of $g_{3,3}$ are

$$
f_{n}=\frac{\left(1-z+z^{3}-z^{4}\right) \pm z^{n-3}\left(1-z+2 z^{3}-z^{4}\right)}{\left(1-2 z+z^{3}-z^{4}\right) \pm z^{n-3}\left(1-z+z^{3}-z^{4}\right)}
$$

for $n \geqslant 23$.

Incorporating the results from [1], we find an interval $\left(\theta_{1}, \theta_{2}\right)$ of $\alpha_{3}$ in which the only elements of $S$ are the roots of $z^{n}\left(z^{4}-2 z^{3}+z-1\right) \pm A(z)$, where $A(z)$ is one of $z^{3}-z^{2}+1, z^{3}-z+1$ or $z^{4}-z^{3}+z-1$. The endpoint $\theta_{1}=1.8667463463$ is an element of $S$ of degree 23 associated with $g_{2,3}$, while $\theta_{2}=1.8667627119$ is an element of $S$ of degree 42 associated with $g_{3,3}$. The minimal polynomials for $\theta_{1}$ and $\theta_{2}$ are (writing $a_{0} z^{d}+a_{1} z^{d-1}+\cdots+a_{d} \equiv a_{0} a_{1} \cdots a_{d}$ ),

$$
\begin{aligned}
& P_{1}=1-1-1-21111-1-10000-10001-1-1-11110-1-1 \text {, } \\
& P_{2}=1-21-1-23-331-24-410-33-2111-11 \\
& -11-110-22-311-24-2111-32-201-11 \text {. }
\end{aligned}
$$

Here $-P_{1}(z) / z^{23} P_{1}\left(z^{-1}\right)$ is in $\mathscr{T}_{17}\left(g_{2,3}\right)$ while $P_{2}(z) / z^{42} P_{2}\left(z^{-1}\right)$ is in $\mathscr{T}_{22}\left(g_{3,3}\right)$.

2. The Derived Tree for $g_{3,3}$. If $f$ is a limit point of $\mathscr{C}$, the derived tree $\mathscr{T}^{\prime}(f)$ consists of all sequences $\left(c_{0}, \ldots, c_{k}\right)$ of integers satisfying the inequalities $c_{0} \neq 0$ and

$$
W_{k}\left(c_{0}, \ldots, c_{k-1}\right) \leqslant c_{k} \leqslant W_{k}^{*}\left(c_{0}, \ldots, c_{k-1}\right),
$$

where $W_{k}$ and $W_{k}^{*}$ are defined by the recurrence relations (4.16) to (4.20) of [2].

Because of the symmetry $W_{k}\left(-c_{0}, \ldots,-c_{k-1}\right)=-W_{k}^{*}\left(c_{0}, \ldots, c_{k-1}\right)$, we may assume $c_{0}>0$. Table 1 gives the values of $W_{k}$ and $W_{k}^{*}$ for $\mathscr{T}^{\prime}=\mathscr{T}^{\prime}\left(g_{3,3}\right)$ truncated to 6 decimal places. The integers in the columns headed $M_{k}$ and $M_{k}^{*}$ will be defined in Section 5. The tree $\mathscr{T}^{\prime}$ is infinite but we have truncated it at nodes where $W_{k}=W_{k}^{*}$ to obtain a finite tree. 
TABLE 1

\begin{tabular}{rrrcrr}
$k$ & $c_{k-1}$ & $W_{k}$ & $W_{k}^{*}$ & $M_{k}$ & $M_{k}^{*}$ \\
\hline 1 & 1 & 1.675282 & 4.045357 & 10 & 11 \\
2 & 2 & 3.898594 & 5.019511 & 18 & 10 \\
3 & 4 & 8.051692 & 8.420621 & 50 & 26 \\
3 & 5 & 11.991607 & 12.068292 & 15 & 16 \\
4 & 12 & 27.970103 & 28 & 21 & 19 \\
5 & 28 & 62 & 62 & 24 & 17 \\
2 & 3 & 6.497327 & 8.834473 & 21 & 31 \\
3 & 7 & 15.421767 & 17 & 34 & 1 \\
4 & 16 & 34.989364 & 36.454884 & 37 & 35 \\
5 & 35 & 76.001155 & 76.043389 & 98 & 43 \\
5 & 36 & 77.788620 & 79.043389 & 41 & 43 \\
6 & 78 & 165.900195 & 166.603279 & 51 & 55 \\
7 & 166 & 347.051834 & 347.394384 & 82 & 60 \\
6 & 79 & 168.824854 & 168.992408 & 50 & 90 \\
4 & 17 & 39 & 39 & 1 & 1 \\
3 & 8 & 17.897284 & 20.043389 & 27 & 27 \\
4 & 18 & 40.426227 & 40.817428 & 45 & 42 \\
4 & 19 & 42.458813 & 44.603279 & 42 & 39 \\
5 & 43 & 94.073654 & 95.692095 & 66 & 44 \\
6 & 95 & 204.179028 & 205.763565 & 66 & 61 \\
7 & 205 & 433.131847 & 434.714303 & 74 & 70 \\
8 & 434 & 904.807006 & 906.374505 & 65 & 74 \\
9 & 905 & 1866.786707 & 1867.463636 & 77 & 77 \\
10 & 1867 & 3816.608989 & 3817.193336 & 88 & 84 \\
11 & 3817 & 7744.362025 & 7744.879502 & 95 & 109 \\
9 & 906 & 1869.373630 & 1870.513744 & 82 & 87 \\
10 & 1870 & 3823.956019 & 3825.085007 & 88 & 91 \\
11 & 3824 & 7761.021063 & 7761.190135 & 127 & 93 \\
11 & 3825 & 7763.546869 & 7763.861294 & 100 & 116 \\
5 & 44 & 96.660124 & 98.394384 & 46 & 44 \\
6 & 97 & 208.959271 & 210.052343 & 55 & 44 \\
7 & 209 & 442.830908 & 442.987753 & 68 & 91 \\
7 & 210 & 445.947206 & 446.146552 & 47 & 58 \\
8 & 446 & 932.819400 & 932.974648 & 61 & 85 \\
6 & 98 & 211.542501 & 212.761293 & 54 & 60 \\
7 & 212 & 449.480521 & 450.623590 & 66 & 59 \\
8 & 450 & 941.125009 & 942.258594 & 84 & 63 \\
9 & 942 & 1947.904856 & 1948.703271 & 72 & 82 \\
10 & 1948 & 3990.600850 & 3990.936074 & 87 & 98 \\
4 & 20 & 45.822361 & 45.992408 & 34 & 74 \\
2 & 4 & 9.822045 & 10 & 18 & 23 \\
3 & 10 & 24 & 24 & 29 & 36 \\
& & & & &
\end{tabular}




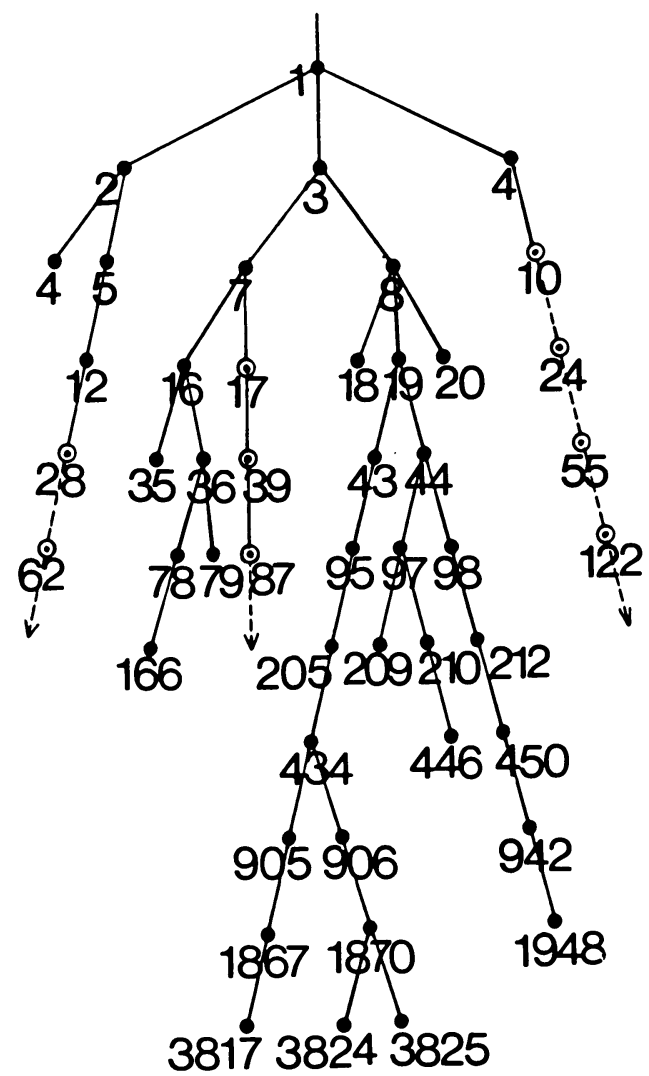

FIGURE 1

The derived tree for $g_{3,3}$

The tree is traversed in left preorder so that listing $c_{k-1}$ suffices to identify a given node $\left(c_{0}, \ldots, c_{k-1}\right)$. A comparison with Figure 1 reproduced from [2], may be helpful. For example, the row of the table giving

$$
c_{4}=35, \quad W_{5}=76.001155, \quad W_{5}^{*}=76.043389
$$

refers to the node $(1,3,7,16,35)$ of $\mathscr{T}^{\prime}$. Since there is no integer $c_{5}$ satisfying $(2.1)$ in this case, $(1,3,7,16,35)$ is a terminal node of $\mathscr{T}^{\prime}$.

The values of $W_{k}^{*}$ listed without fractional part are exactly integers. The branches of $\mathscr{T}^{\prime}$ corresponding to $c_{k}=W_{k}^{*}$ are simple paths to infinity in $\mathscr{T}^{\prime}(f)$ discussed more fully in [2, Section 9].

The trees $\mathscr{T}_{n}$ consist of all $\left(c_{0}, \ldots, c_{k}\right)$ for which $\left(u_{0}, \ldots, u_{n-1}, u_{n}+c_{0}, \ldots, u_{n+k}+\right.$ $c_{k}$ ) are in $\mathscr{T}$. (Here $g_{3,3}=u_{0}+u_{1} z+\cdots$.) These trees are characterized by inequalities similar to (2.1):

$$
W_{n, k}\left(c_{0}, \ldots, c_{k-1}\right) \leqslant c_{k} \leqslant W_{n, k}^{*}\left(c_{0}, \ldots, c_{k-1}\right) .
$$

We will use $\mathscr{T}_{n}^{+}\left(\mathscr{T}_{n}^{-}\right)$to refer to the sequences satisfying $(2.3)$ and $c_{0}>0\left(c_{0}<0\right)$ respectively.

By Theorem 7.3 of [2], there are effective constants $N_{k}$ such that, for $n \geqslant N_{k}$, each path $\left(c_{0}, \ldots, c_{k}\right)$ in $\mathscr{T}_{n}(f)$ is also a path in $\mathscr{T}^{\prime}(f)$. This depends on the convergence $W_{n, k} \rightarrow W_{k}$ and $W_{n, k}^{*} \rightarrow W_{k}^{*}$ as $n \rightarrow \infty$. 
As Figure 1 shows, the effective height of $\mathscr{T}^{\prime}\left(g_{3,3}\right)$ is 10 and hence we need only estimate $N_{11}$. For this, it suffices to find an $N$ such that, for $n \geqslant N$, and $\left(c_{0}, \ldots, c_{k-1}\right)$ a node in $\mathscr{T}^{\prime}$ with $k-1 \leqslant 10$, we have

$$
\left[W_{n, k}, W_{n, k}^{*}\right] \cap \mathbf{Z} \subseteq\left[W_{k}, W_{k}^{*}\right] \cap \mathbf{Z} .
$$

Inspection of Table 1 suggests that the most stringent requirement here is that $76<W_{n, 5}<77$ for the node $(1,3,7,16,35)$ of $(2.2)$. This is indeed the case and we will show that $N_{5}=N_{6}=\cdots N_{11}=63$. Thus $\mathscr{T}_{n}^{ \pm}(f), n \geqslant 63$ has only one path to infinity, the "regular" path corresponding to $f_{n}$ of (1.1). In fact this is true for $n \geqslant 23$ even though $\mathscr{T}_{n}$ in this case usually contains extraneous nodes which are not in the tree $\mathscr{T}^{\prime}$. The tree $\mathscr{T}_{22}^{+}$has two paths to infinity, the regular path and one corresponding to (1.3). The other $\mathscr{T}_{n}$ for small $n$ are discussed more fully in Section 6 .

3. Formulae for Estimating $W_{n, k}-W_{k}$. To estimate $N_{k}$, we will need estimates for $W_{n, k}-W_{k}$ and $W_{n, k}^{*}-W_{k}^{*}$. We develop estimates in detail only for $W_{n, k}-W_{k}$ since the treatment of $W_{n, k}^{*}-W_{k}^{*}$ is almost identical.

According to $[2,(7.5)$ and (7.6)] we have

$$
\frac{\Omega}{Q^{2}} \frac{P_{n, k}}{Q_{n, k}}=c_{0}+\cdots+c_{k-1} z^{k-1}+W_{n, k} z^{k}+\cdots \quad(n>k+1)
$$

and

$$
\frac{\Omega}{Q^{2}} \frac{G d_{k}}{\tilde{G} e_{k}}=c_{0}+\cdots+c_{k-1} z^{k-1}+W_{k} z^{k}+\cdots .
$$

Here $\Omega=\left(1-z+z^{3}\right)\left(1-z^{2}+z^{3}\right), Q=1-2 z+z^{3}-z^{4}, Q_{n, k}$ is of degree $k+3$ with $Q_{n, k}(0)=1$ and $P_{n, k}(z)=-z^{k+3} Q_{n, k}\left(z^{-1}\right)$. Also $\Omega(z)=$ $G(z) \tilde{G}(z) / G(0)$, where $G$ is monic, of degree 3 , and has all its zeros in $|z|>1$, while $\tilde{G}(z)=z^{3} G\left(z^{-1}\right)$. The polynomials $d_{k}, e_{k}$ satisfy $e_{k}(0)=1, \operatorname{deg} e_{k}=k$ and $d_{k}=$ $-z^{k} e_{k}\left(z^{-1}\right)$.

If $c_{m-1} \neq W_{m-1}$ or $W_{m-1}^{*}$ for $m \leqslant k$ then these conditions and (3.2) characterize $d_{k}$ and $e_{k}$. If $c_{m-1}=W_{m-1}$ or $W_{m-1}^{*}$ for some $m \leqslant k$ then we define $e_{k}(z)=$ $(1+z)^{k-m} e_{m}(z)$. In this case $c_{0}, \ldots, c_{m-1}$ uniquely determine $c_{m}, c_{m+1}, \ldots$ Similar remarks apply to $P_{n, k}$ and $Q_{n, k}$ if $n>k+1$.

LEMMA 3.1. With the above notation, if $n>k+1$, then

$$
P_{n, k} \tilde{G} e_{k}-Q_{n, k} G d_{k}=z^{k} H_{n, k},
$$

where $H_{n, k}$ is a polynomial of degree at most $2 r$ which satisfies $z^{2 r} H_{n, k}\left(z^{-1}\right)=-H_{n, k}(z)$, and

$$
W_{n, k}-W_{k}=H_{n, k}(0) \text {. }
$$

Proof. The left member of (3.3) is a polynomial $R$ of degree at most $2 k+2 r$ which satisfies

$$
z^{2 k+2 r} R\left(z^{-1}\right)=-R(z)
$$

By (3.1) and (3.2), it follows that

$$
\Omega R /\left(Q^{2} Q_{n, k} \tilde{G} e_{k}\right)=\left(W_{n, k}-W_{k}\right) z^{k}+\cdots,
$$

so $R$ has a zero of order $k$ at $z=0$. Thus $R(z)=z^{k} H_{n, k}(z)$ where $\operatorname{deg} H_{n, k} \leqslant k+2 r$. But by (3.5) the leading $k$ coefficients of $r$ also vanish so, in fact, deg $H_{n, k} \leqslant 2 r$. The remaining properties of $H_{n, k}$ follow from (3.5) and (3.6).

For our example, $r=3$ so that

$$
H_{n, k}(z)=a+b z+c z^{2}-c z^{4}-b z^{5}-a z^{6},
$$


where $a=W_{n, k}-W_{k}$. Let $\alpha_{i},(i=1,2,3)$, be the zeros of $G$ so $\alpha_{1}$ is the real root of $1-z+z^{3}=0$ and $\alpha_{2}, \alpha_{3}$ the complex roots of $1-z^{2}+z^{3}$. Numerically,

$$
\alpha_{1}=-1.3247179572, \quad \alpha_{2}=.8774388331+i(.7448617666) \text {. }
$$

From (3.3), we have

$$
H_{n, k}\left(\alpha_{i}\right)=\alpha_{i}^{-k} P_{n, k}\left(\alpha_{i}\right) \tilde{G}\left(\alpha_{i}\right) e_{k}\left(\alpha_{i}\right) \quad(i=1,2,3) .
$$

From (3.4) we have, for $n>k+1$,

$$
W_{n, k}-W_{k}=\sum_{i=1}^{3} h_{i} H_{n, k}\left(\alpha_{i}\right),
$$

where $h_{1}, h_{2}$ and $h_{3}$ are obtained by solving the following $3 \times 3$ linear system for $a$ :

$$
a\left(1-\alpha_{i}^{6}\right)+b\left(\alpha_{i}-\alpha_{i}^{5}\right)+c\left(\alpha_{i}^{2}-\alpha_{i}^{4}\right)=H_{n, k}\left(\alpha_{i}\right) .
$$

Numerically,

$$
h_{1}=.057477943, \quad\left|h_{2}\right|=\left|h_{3}\right|=.374123681 .
$$

The quantities $\alpha_{i}^{k} \tilde{G}\left(\alpha_{i}\right) e_{k}\left(\alpha_{i}\right)$ can be computed for each node of the derived tree. The quantities $P_{n, k}\left(\alpha_{i}\right)$ tend to zero geometrically. So Formulae (3.8) and (3.9) provide a means of estimating $W_{n, k}-W_{k}$. It is useful, though, to further manipulate (3.8).

LEMMA 3.2. With the above notation,

$$
P_{n, k} Q_{n, k+1}-Q_{n, k} P_{n, k+1}=z^{k}(1-z)\left(W_{n, k}-c_{k}\right) \Omega \text {. }
$$

Proof. Combine the definitions [2, (5.6) and (5.10)] of $P_{n, k}$ and $Q_{n, k}$ with the following formula from $[3$, p. 82],

$$
D_{n+1} E_{n}-D_{n} E_{n+1}=\left(u_{n}-w_{n}\right) z^{n}(1-z) \text {. }
$$

Corollary 3.3. Let $f=g_{3,3}$. Then, for all $n, k$ and $i=1,2,3$,

$$
P_{n, k}\left(\alpha_{i}\right)=\alpha_{i}^{-1-n} Q_{n, k}\left(\alpha_{i}\right) \text {. }
$$

Proof. By (3.12), the ratio $P_{n, k}\left(\alpha_{i}\right) / Q_{n, k}\left(\alpha_{i}\right)$ is independent of $k$. When $k=0$, $P_{n, k}=P_{n}$ and $[2,(5.9)]$ gives

$$
z^{n+r} P_{n}(z) A\left(z^{-1}\right)-z^{r} P_{n}\left(z^{-1}\right) Q(z)=E_{n}(z) \Omega(z) .
$$

Thus

$$
P_{n}\left(\alpha_{i}\right) / P_{n}\left(\alpha_{i}^{-1}\right)=\alpha_{i}^{-n} Q\left(\alpha_{i}\right) / A\left(\alpha_{i}^{-1}\right) .
$$

For $f=g_{3,3}$ we readily verify that

$$
Q(z)+z^{2} A\left(z^{-1}\right)=-z^{-2} \Omega(z),
$$

and hence $Q\left(\alpha_{i}\right) / A\left(\alpha_{i}^{-1}\right)=-\alpha_{i}^{2}$. Since $Q_{n, 0}(z)=-z^{3} P_{n}\left(z^{-1}\right)$, we can now derive (3.13) for $k=0$ and hence for all $k$.

The formula (3.8) can thus be written in the more useful form:

$$
H_{n, k}\left(\alpha_{i}\right)=\alpha_{i}^{-n-k-1} \tilde{G}\left(\alpha_{i}\right) e_{k}\left(\alpha_{i}\right) Q_{n, k}\left(\alpha_{i}\right)
$$

It only remains to estimate $Q_{n, k}\left(\alpha_{i}\right)$, to which we now turn.

4. Estimates for the Auxiliary Polynomials. Throughout this section, $P_{n, k}$ etc. refer to the auxiliary polynomials for $f=g_{3,3}$. The techniques apply to any $f$ for which $\Omega$ has no multiple zeros. We regard polynomials of degree $d$ with real coefficients as 
vectors in $\mathbf{R}^{d+1}$ and measure distance between two such polynomials by the $l^{\infty}$-norm, i.e., $\left\|a_{0} x^{d}+\cdots+a_{d}\right\|=\max \left\{\left|a_{i}\right|: 1 \leqslant i \leqslant d\right\}$. Recall that $P_{n, 0}=P_{n}$ and $Q_{n, 0}=Q_{n}$.

LEMMA 4.1. For $n \geqslant 50$,

$$
\left\|P_{n}-G\right\|=\left\|Q_{n}-\tilde{G}\right\| \leqslant C \delta^{n} \leqslant .007,
$$

where $C \leqslant 7.871$ and $\delta=\left|\alpha_{2}\right|^{-1} \leqslant .868837$.

Proof. Temporarily let

$$
G(z)=\left(z-\alpha_{1}\right)\left(z-\alpha_{2}\right)\left(z-\alpha_{3}\right)=a+b z+c z^{2}+z^{3}
$$

and

$$
P_{n}(z)=a_{n}+b_{n} z+c_{n} z^{2}+z^{3}
$$

From (3.13) we have

$$
P_{n}\left(\alpha_{i}\right)=-\alpha_{i}^{2-n} P_{n}\left(\alpha_{i}^{-1}\right)
$$

Thus

$$
a+b \alpha_{i}+c \alpha_{i}^{2}=-\alpha_{i}^{3}
$$

and

$$
a_{n}\left(1+\alpha_{i}^{2-n}\right)+b_{n}\left(\alpha_{i}+\alpha_{i}^{1-n}\right)+c_{n}\left(\alpha_{i}^{2}+\alpha_{i}^{-n}\right)=-\alpha_{i}^{3}-\alpha_{i}^{-1-n} .
$$

Define the $3 \times 3$ matrices $V$ and $U_{n}$ to have rows $\left(1, \alpha_{i}, \alpha_{i}^{2}\right)$ and $\left(\alpha_{i}^{2-n}, \alpha_{i}^{1-n}, \alpha_{i}^{-n}\right)$ respectively, and define the column vectors $p_{n}, g$ and $h_{n}$ by $p_{n}=\left(a_{n}, b_{n}, c_{n}\right)^{\text {tr }}$, $g=(a, b, c)^{\mathrm{tr}}$ and $h_{n}=\left(-\alpha_{1}^{-1-n},-\alpha_{2}^{-1-n},-\alpha_{3}^{-1-n}\right)^{\mathrm{tr}}$. Then (4.4) and (4.5) combine into

$$
\left(V-U_{n}\right) p_{n}=V g+h_{n},
$$

or

$$
p_{n}-g=\left(I-V^{-1} U_{n}\right)^{-1} V^{-1}\left(U_{n} g+h_{n}\right) .
$$

Using the $l^{\infty}$-norm, we calculate

$$
\begin{aligned}
& \left\|V^{-1}\right\|=1.125642247, \\
& \left\|U_{n}\right\|=(3.475681885) \delta^{n}, \quad n \geqslant 2, \\
& \left\|h_{n}\right\|=\delta^{1+n} \\
& \|g\|=\alpha_{1}^{2}=1.754877666,
\end{aligned}
$$

so that $\left(1-\left\|V^{-1} U_{n}\right\|\right)^{-1} \leqslant 1.00347425$ for $n \geqslant 50$. Hence (4.7) gives

$$
\left\|p_{n}-g\right\| \leqslant\left(1-\left\|V^{-1} U_{n}\right\|\right)^{-1}\left\|V^{-1}\right\|\left(\left\|U_{n}\right\|\|g\|+\left\|h_{n}\right\|\right),
$$

which proves the lemma.

Lemma 4.2. For $n \geqslant 51$,

$$
\left|Q_{n, k}\left(\alpha_{i}\right)\right| \leqslant C_{i} \rho_{i}^{k}
$$

where

$$
C_{1}=4.302463189, \quad C_{2}=C_{3}=1.204779188,
$$


and where $\rho_{i}$ is the larger root of $x^{2}=\left|1+\alpha_{i}\right| x+4\left|\alpha_{i}\right|$, so that

$$
\rho_{1}=2.470005456, \quad \rho_{2}=\rho_{3}=3.381346122 \text {. }
$$

Proof. From [2, (5.19)], we deduce that

$$
Q_{n, k+1}=(1+z) Q_{n, k}-\gamma_{n, k} z Q_{n, k-1},
$$

where $\gamma_{n, k}=\left(c_{k}-W_{n, k}\right) /\left(c_{k-1}-W_{n, k-1}\right)$. From [2, (5.21)], we have

(4.11) $W_{n, k}^{*}-W_{n, k}=4\left(W_{n, k-1}^{*}-c_{k-1}\right)\left(c_{k-1}-W_{n, k-1}\right) /\left(W_{n, k-1}^{*}-W_{n, k-1}\right)$.

Combining (2.3) and (4.11) yields $0 \leqslant \gamma_{n, k} \leqslant 4$, provided $c_{k-1} \neq W_{n, k-1}$. (If $c_{k-1}=$ $W_{n, k-1}$ then $Q_{n, m}$ for $m \geqslant k+1$ is not uniquely defined, but if we simply let $Q_{n, m}=(1+z)^{m-k} Q_{n, k}$ in this case, then (4.10) holds with $0 \leqslant \gamma_{n, k} \leqslant 4$.)

The initial conditions in (4.10) are

$$
Q_{n, 0}=Q_{n} \text { and } Q_{n,-1}=Q_{n-1} \text {. }
$$

If we demonstrate (4.9) for $k=0,1$ it will follow by induction for $k \geqslant 2$ from (4.10) and $\left|\gamma_{n, k}\right| \leqslant 4$. By Lemma 4.1, $\left\|Q_{n}-\tilde{G}\right\| \leqslant .007$ for $n \geqslant 50$, so

$$
\left|Q_{n}\left(\alpha_{i}\right)\right| \leqslant\left|\tilde{G}\left(\alpha_{i}\right)\right|+(.007)\left(\left|\alpha_{i}\right|+\left|\alpha_{i}\right|^{2}+\left|\alpha_{i}\right|^{3}\right) \text {. }
$$

Since

$$
\tilde{G}\left(\alpha_{1}\right)=-4.264632994 \text { and }\left|\tilde{G}\left(\alpha_{2}\right)\right|=1.176776497,
$$

we do have (4.9) for $k=0$ and $i=1,2,3$.

To estimate $Q_{n, 1}\left(\alpha_{i}\right)$, we use (4.10) and (4.12) where

$$
\gamma_{n, 0}=\left(c_{0}+u_{n}-w_{n}\right) /\left(u_{n-1}-w_{n-1}\right)
$$

and $c_{0}= \pm 1$. From Lemma 4.1

$$
\left|\left(u_{n}-w_{n}\right)-w\right| \leqslant\left\|P_{n}-G\right\| \leqslant .007 \text { if } n \geqslant 50 .
$$

Hence $\left|\gamma_{n, 0}-\gamma_{0}\right| \leqslant .010291843$, for $n \geqslant 51$, where $\gamma_{0}=\left(c_{0}+w\right) / w=1.569840291$ (if $c_{0}=1$ ) or $\gamma_{0}=.430159709$ (if $c_{0}=-1$ ).

Now write

$$
\begin{aligned}
Q_{n, 1}= & (1+z) \tilde{G}-\gamma_{0} z \tilde{G}+(1+z)\left(Q_{n}-\tilde{G}\right)-\gamma_{n, 0} z\left(Q_{n-1}-\tilde{G}\right) \\
& -\left(\gamma_{n, 0}-\gamma_{0}\right) z \tilde{G},
\end{aligned}
$$

and use the estimates for $\left|\gamma_{n, 0}-\gamma_{0}\right|$ and $\left\|Q_{n}-\tilde{G}\right\|$ given above to verify (4.9) for $k=1, n \geqslant 51$. This completes the proof of the lemma.

Remark. The estimate (4.9) is rather unrealistic for large $k$ since we know [2, Lemma 7.1] that $Q_{n, k} \rightarrow \tilde{G} e_{k}$ as $n \rightarrow \infty$ at interior nodes of the derived tree (nodes where $\left.W_{k-1}<c_{k-1}<W_{k-1}^{*}\right)$. The estimate can be improved by recursively estimating the differences $\gamma_{n, k}-\gamma_{k}$ and using the known values of $\gamma_{k}$, rather than resorting to the estimate $0 \leqslant \gamma_{n, k} \leqslant 4$. This turns out to be unnecessary for our purposes.

5. Estimates of $N_{k}$. Combining (3.9), (3.11), (3.17) and (4.9), at each node of the derived tree we have

$$
\left|W_{n, k}-W_{k}\right| \leqslant \sum_{i=1}^{3} A_{k, i}\left|e_{k}\left(\alpha_{i}\right)\right|\left|\alpha_{i}\right|^{-n},
$$

where $A_{k, i}=\left|h_{i}\right| C_{i}\left|\alpha_{i}\right|^{-1-k} \rho_{i}^{k}$, so that

$$
A_{k, 1}=(.796116505)(1.864551955)^{k} \text {, }
$$




$$
A_{k, 2}=A_{k, 3}=(.460845058)(2.937838492)^{k} .
$$

Tracing through the analysis, we find that Lemmas 4.1 and 4.2 are valid for $P_{n, k}^{*}$ and hence that

$$
\left|W_{n, k}^{*}-W_{k}^{*}\right| \leqslant \sum_{i=1}^{3} A_{k, i}\left|e_{k}^{*}\left(\alpha_{i}\right)\right|\left|\alpha_{i}\right|^{-n} .
$$

Let $U_{k}$ be the largest integer strictly smaller than $W_{k}$ and $V_{k}^{*}$ be the smallest integer strictly larger than $W_{k}^{*}$, so

$$
\begin{array}{ll}
W_{k}=U_{k}+\delta_{k}, & 0<\delta_{k} \leqslant 1, \\
W_{k}^{*}=V_{k}^{*}-\delta_{k}^{*}, & 0<\delta_{k}^{*} \leqslant 1 .
\end{array}
$$

For each node in the derived tree, let $m_{k}=m_{k}\left(c_{0}, \ldots, c_{k-1}\right)$ and $m_{k}^{*}$ be such that $m \geqslant m_{k}$ implies $\left|W_{n, k}-W_{k}\right|<\delta_{k}$ and $m>m_{k}^{*}$ implies $\left|W_{n, k}^{*}-W_{k}^{*}\right|<\delta_{k}^{*}$. If $M_{k}$ $\left(M_{k}^{*}\right)$ are such that the right members of (5.1) and (5.4) are less than $\delta_{k}\left(\delta_{k}^{*}\right)$ for $m \geqslant M_{k}\left(M_{k}^{*}\right)$, then $m_{k} \leqslant \max \left(M_{k}, 51\right)$ and $m_{k}^{*} \leqslant \max \left(M_{k}^{*}, 51\right)$. The quantities $M_{k}$ and $M_{k}^{*}$ are given in Table 1.

Suppose $\left(c_{0}, \ldots, c_{k-1}\right)$ is a path in $\mathscr{T}^{\prime}$ and in $\mathscr{T}_{n}$. Then, for $n \geqslant \max \left(m_{k}, m_{k}^{*}\right)$, (2.4) holds. As shown in [2, Theorem 8.4(b)], no path in $\mathscr{T}_{n}$ can follow any of the paths to infinity in $\mathscr{T}^{\prime}$ to a height $>4$ unless it coincides with one of $\pm(1,3,7,17,39, \ldots)$. Thus if $\mathscr{T}^{\prime}$ is truncated at height 4 on these paths and $N=\max \left(m_{k}, m_{k}^{*}\right)$ over all nodes in the truncated $\mathscr{T}^{\prime}$ then $n \geqslant N$ implies that $\mathscr{T}_{n}$ is a subtree of $\mathscr{T}^{\prime}$.

The bounds $M_{k}, M_{k}^{*}$ given in Table 1 together with values for the nodes where $c_{k}=W_{k}^{*}$ and $k \leqslant 4$ give $N \leqslant 127$. By computing $\mathscr{T}_{n}$ for $n \leqslant 126$ as described in the next section, we obtain exact values for $m_{k}$ and $m_{k}^{*}$. For example,

$$
m_{5}(1,3,7,16,35)=63 \text { and } m_{11}(1,3, \ldots, 1870,3824)=47 .
$$

If we use $Q_{n, k}\left(\alpha_{i}\right) \approx \tilde{G}\left(\alpha_{i}\right) e_{k}\left(\alpha_{i}\right)$ rather than (4.9) we obtain $m_{5}(1, \ldots, 35) \approx 66$ and $m_{11}(1, \ldots, 3824) \approx 48$, so we see that it is (4.9) that leads to the more pessimistic values 98 and 127 given in Table 1 for these two quantities.

For the sake of interest,

$$
W_{62.5}^{*}(-1,-3,-7,-16,-35)=75.999171,
$$

and

$$
W_{46.11}(1,3, \ldots, 1870,3824)=7760.998730,
$$

correct to 6 decimal places.

6. The Trees $\mathscr{T}_{n}\left(g_{3,3}\right)$. By the calculations of the previous section, if $n \geqslant 127$, each tree $\mathscr{T}_{n}{ }^{ \pm}\left(g_{3,3}\right)$ contains only the single path to infinity $\pm(1,3,7,17, \ldots)$ corresponding to $f_{n}$ of (1.1). It thus remains to examine the trees $\mathscr{T}_{n}{ }^{ \pm}$for $n<127$ to determine the finite number of exceptional elements of $\mathscr{C}$ in the neighborhood of $g_{3,3}$.

In the following discussion of the sizes of $\mathscr{T}_{n}{ }^{ \pm}$, we have truncated these trees at the nodes where $c_{k}=W_{n, k}$ or $W_{n, k}^{*}$. Since the expansion of $g_{3,3}$ begins

$$
g_{3,3}=1,1,2,4,7,13,24,45, \ldots
$$

it is clear that $\left|\mathscr{T}_{4}^{+}\right|=\infty$ since it contains a path corresponding to

$$
(1-z) /(1-2 z)=1,1,2,4,8, \ldots
$$

which is in the second derived set of $\mathscr{C}$. 
Similarly, $\left|\mathscr{T}_{5}^{+}\right|=\infty$ since the limit point $\beta_{3}=1.927 \ldots$ has an expansion

$$
\left(1-z^{4}\right) /\left(1-z-z^{2}-z^{3}-z^{4}\right)=1,1,2,4,7,14, \ldots .
$$

All other $\mathscr{T}_{n}^{ \pm}$with $n \geqslant 4$ are finite. Although both of $\mathscr{T}_{6}^{ \pm}$are small, $\mathscr{T}_{7}^{-}$is quite large, containing 1671 nodes and 102 paths corresponding to elements of $\mathscr{C}$. These expansions begin

$$
1,1,2,4,7,13,24,44, \ldots
$$

and the corresponding Pisot numbers lie in a rather small neighborhood of $\beta_{2}=$ $1.839 \ldots$ rather than of $\alpha_{3}=1.866 \ldots$ and are among those discussed at the end of Section 3 of [1].

For $n \geqslant 23$, there are no paths to infinity other than those corresponding to (1.1). For $n=22$, we obtain $P_{2}$ of $(1.2)$ corresponding to a path $(1,3,8, \ldots, 3682615)$ in $\mathscr{T}_{22}^{+}$.

In spite of the restriction $n>k+1$, in (3.1), the trees $\mathscr{T}_{n}^{ \pm}$can be computed correctly for all $n$ by using (3.1). That is, $P_{n, k}$ and $Q_{n, k}$ are computed from (4.10) and $W_{n, k}$ from (3.1), and similarly with $P_{n, k}^{*}, Q_{n, k}^{*}$. If $k+1 \geqslant n$ then the values for $c_{k}$, $W_{n, k}$ and $W_{n, k}^{*}$ are incorrect, but the quantities $c_{k}-W_{n, k}$ and $W_{n, k}^{*}-c_{k}$ are correct, so the recurrence relation (4.10) gives the correct $P_{n, k}$ and $Q_{n, k}$.

The starting values $P_{n}$ and $P_{n}^{*}$ are computed using the recurrence relations [2, (5.18)]. This was done in integer arithmetic and, for comparison, using double precision floating-point arithmetic. The floating-point calculation proves to be accurate to over 13 decimal places indicating the exceptional stability of the recurrence relation $[2,(5.18)]$.

For $n \leqslant 65$, the trees $\mathscr{T}_{n}^{ \pm}$were computed using integer arithmetic as in [1]. For $n>65$, the computations were done in the double precision floating-point arithmetic. Since the trees in this case are of height at most 11 , it is easy to see that the rounding error gives values of $W_{n, k}$ and $W_{n, k}^{*}$ correct to at least 5 decimal places even under the pessimistic (and false) assumption that $\gamma_{n, k}=4$ for all $n, k$.

The integer calculations for $n \leqslant 65$ make the detection of $P_{n, k}$ and $P_{n, k}^{*}$ with integer coefficients easy. These correspond to $D_{n+k}$ and $D_{n+k}^{*}$ whose roots are Pisot numbers according to the formulae [2, (5.8) and (5.13)]. A comparison with the results of [1] revealed that the only Pisot number in the range [1,1.932] actually missed in [1] was $\theta_{2}$, the root of $P_{2}$ of (1.3).

The computations of this section were performed on an AMDAHL 470/V8. The numbers in Table 1 were computed on an Apple II+ and verified by a computation on the AMDAHL 470/V8. In fact, all of the computations described here are well within the capabilities of a microcomputer except for the computation of some of the larger $\mathscr{T}_{n}$ such as $\mathscr{T}_{7}^{-}$.

Department of Mathematics University of British Columbia Vancouver, B. C., Canada V6T 1Y4

1. D. W. Boyd, "Pisot and Salem numbers in intervals of the real line," Math. Comp., v. 32, 1978, pp. 1244-1260.

2. D. W. BoYD, "Pisot numbers in the neighbourhood of a limit point. I," (To appear.)

3. J. Dufresnoy \& CH. Pisot, "Étude de certaines fonctions méromorphes bornées sur le cercle unité, application à un ensemble fermé d'entiers algébriques," Ann. Sci. École Norm. Sup. (3), v. 72, 1955, pp. $69-92$. 